\title{
circRNA-miRNA association for coronary heart disease
}

\author{
FEI LIN, GUOAN ZHAO, ZHIGANG CHEN, XUEHUI WANG, FENGHUA LV, YONGCHUN ZHANG, \\ XIAODONG YANG, WANQIAN LIANG, RUIYAN CAI, JIANHUA LI, MENG LI and GUHAO ZHANG
}

\author{
Cardiovascular Research Center, The First Affiliated Hospital of Xinxiang Medical University, \\ Xinxiang, Henan 453100, P.R. China
}

Received January 4, 2018; Accepted January 8, 2019

DOI: $10.3892 / \mathrm{mmr} .2019 .9905$

\begin{abstract}
Coronary heart disease (CHD) is a major cause of morbidity and mortality and an important public health problem globally, but the mechanism of CHD is still complex and unclear. The purpose of the current study was to explore the mechanism underlying CHD using high-throughput technology. The study participants were patients with coronary angiography (CAG)-proven severity of coronary artery stenosis. Patients were divided into control and test group based on specific inclusion criteria, and data were collected regarding the results of routine inspection and the Gensini score (GS). We explored the mechanism underlying CHD with high-throughput integration of circular RNA (circRNA)-microRNA (miRNA) data. Through the expression of circRNA-miRNA, we discovered a total of 110 circRNAs to be differentially expressed in the two groups. Of these, 73 were upregulated and 37 downregulated in the CHD (fold $\geq 2.0$ and $\mathrm{P}<0.05$ ). Among 18 miRNAs, 13 were upregulated and 5 were downregulated in the CHD group (fold $\geq 2.0$ and $\mathrm{P}<0.05$ ). Enrichment analysis showed that circRNAs participate in a variety of disease development processes, biological processes, molecular functions, cellular components, and pathways $(\mathrm{P}<0.05)$. The mechanism underlying CHD may be closely related to up- or downregulated circRNA and miRNA and co-expression of circRNA-miRNA specifically involved regulate multiple pathways and multiple cellular and molecular biological processes.
\end{abstract}

\section{Introduction}

Coronary heart disease (CHD) brings severe health problems to individuals. CHD prevention is of considerable importance for patients and medical staff. With the development of high-throughput sequencing and bioinformatics, it has been found that circular RNA (circRNA) enriches other types

Correspondence to: Dr Guoan Zhao, Cardiovascular Research Center, The First Affiliated Hospital of Xinxiang Medical University, 88 Jiankang Road, Weihui, Xinxiang, Henan 453100, P.R. China

E-mail: zhaoguoan1706@163.com

Key words: coronary heart disease, atherosclerosis, circular RNA, microRNA, high-throughput sequencing of non-coding RNA (ncRNA). circRNA is a novel type of ncRNA which could be found within mammalian cells, and may have been an overlooked feature of eukaryotic gene expression and regulation $(1,2)$. circRNA is plentiful and stable in exosomes, and an increasing number of its functions have been discovered with the development of modern technology. To date, the functions of circRNA include roles such as microRNA (miRNA) sponges, splicing or transcriptional regulators, and agents interacting with RNA binding proteins (RBPs), influencing the physiological process of aging, insulin secretion, and tissue development. circRNAs also participate in the development of atherosclerotic lesions, neurological disorders, cardiac fibroblasts, cardiac hypertrophy, cancer, and modulating stress and senescence responses (3-5). They may be suitable biomarkers for cancer and coronary artery disease diagnosis, as well as other purposes $(6,7)$.

Collectively, it has been reported that circRNAs might play crucial roles in fundamental life processes and serve as novel clinical molecular markers. They might provide new insights into the prediction, diagnosis, and treatment of diseases and the rehabilitation of patients. It has been reported that circRNAs are expressed in various cardiovascular diseases, such as heart failure and pathological hypertrophy, myocardial infarction, cardiac senescence and atherosclerosis (8). There are a great many circRNAs highly expressed in the heart. There are a total of 15,318 cardiac circRNAs in humans (9). The specific circRNAs hsa_circ_0124644 and hsa_circ_0098964 are significantly upregulated in CHD and can potentially be used as diagnostic markers in CHD (7).

In general, we hypothesized that the development of CHD has some connection with circRNAs, we studied the circRNA-miRNA association for CHD, and compared the peripheral blood circRNA profiles of large independent cohorts of CHD patients and matched control subjects through retrospective and microarray analysis. The results revealed that the mechanism of degree of coronary artery stenosis in patients with CHD may involve mutually regulatory circRNAs and miRNAs or several circRNAs and miRNAs involved in many pathways, cell and molecular biological processes, and molecular features.

\section{Patients and methods}

Study population

Study cohorts. In this study, a total of 40 inpatients were recruited, between November 2016 to February 2017, 
Table I. Coronary Gensini score.

\begin{tabular}{lrlr}
\hline Degree of coronary stenosis & Score & \multicolumn{1}{c}{ Lesion location } & Score \\
\hline $1-25 \%$ & 1 & Left main coronary artery & 5.0 \\
$26-50 \%$ & 2 & Left anterior descending branch or proximal segment of circumflex branch & 2.5 \\
$51-75 \%$ & 4 & Middle left anterior descending branch & 1.5 \\
$76-90 \%$ & 8 & Distal segment of left anterior descending branch & 1.0 \\
$91-99 \%$ & 16 & Left circumflex branch middle and distal segment & 1.0 \\
Full closed & 32 & Right coronary artery & 1.0 \\
& & Small branch & 0.5 \\
\hline
\end{tabular}

Each integral lesion is scored by the degree of stenosis multiplied by the number of lesions, and each patient's score is the sum of all lesions scores.

from the First Affiliated Hospital of Xinxiang Medical University (Xinxiang, China). The age range of patients was 35-74 years. The study was approved by the Ethics Committee of the First Affiliated Hospital of Xinxiang Medical University. Signed written informed consents were obtained from the patients and/or guardians. All participants were examined by coronary angiography (CAG) to verify the presence of CHD. Inclusion criteria: for the test group $(n=20)$, patients with CHD (as verified by CAG) and Gensini score (GS)>40 were included, according to the diagnostic criteria of CHD (I25.105) based on the International Classification of Diseases 10th edition (ICD10); for the control group $(n=20)$, patients with $\mathrm{CHD}$ and $\mathrm{GS}<3$ were included.

Exclusion criteria. Subjects were excluded according to the following criteria: i) with chest pain caused by severe congestive heart failure, dilated cardiomyopathy, severe neurosis, severe arrhythmia, menopause syndrome, hyperthyroidism, gallbladder heart syndrome, stomach and esophageal regurgitation, hiatal hernia, aortic dissection, cervical spondylosis; ii) with liver and kidney dysfunction; iii) suffering from any other clinically systemic acute or chronic inflammatory disease; iv) autoimmune disease; v) uncontrolled hypertension; vi) malignant arrhythmias or valvular heart disease; and vii) malignancy.

Data collection. The basic information of patients came from the hospital medical records. Data were collected concerning age, sex, heart rate (HR), blood pressure (BP), fasting blood glucose (FBG), total cholesterol (CHO), triglycerides (TG), apolipoprotein A1 (APOA1), apolipoprotein B (APOB), high-density lipoprotein (HDL), low-density lipoprotein (LDL), lipoprotein (a) [LP(a)], ejection fraction (EF), and fractional shortening (FS).

The radial CAGs were read by two experts from the Cardiovasology based on the GS (Table I).

\section{Microarray analysis of circRNA-miRNA}

Fabrication of DNA microarray. High-throughput data integration of circRNA-miRNA provided insight into the mechanisms underlying CHD. CircRNA Array v2 (CapitalBio, Corp., Beijing, China) was designed with four identical arrays per slide ( $4 \times 180 \mathrm{~K}$ format), with each array containing probes for $\sim 170,340$ human circRNAs. Those circRNA target sequences were all from circBase (http://www.circbase.org/), deepBase (http://rna.sysu.edu.cn/deepBase/browser.php), and the study of You et al in 2015 (10). Each circRNA was simultaneously detected using a long and a short probe. The circRNA array also contained 4,974 Agilent control probes. Samples were analyzed using Agilent Human miRNA Microarray chips (8x60 K) (release 21.0; Agilent Technologies, Inc., Santa Clara, CA, USA). The raw data were normalized by Quantile algorithm using GeneSpring Software v12.6 (Agilent Technologies, Inc.). The differentially expressed miRNAs that showed a $\geq 2$-fold change were screened.

RNA extraction and fabrication of DNA microarray were performed by the CapitalBio Corp.

Test group and collection of whole blood samples. The patient blood sample collection was performed as follows: $2 \mathrm{ml}$ of blood were collected from the peripheral blood samples of all patients after CAG and then stored in anticoagulant vacutainers treated with EDTA. Then, $750 \mu 1$ TRI pure LS Reagent (RP001; BioTeke Corp., Beijing, China) was added to $250 \mu \mathrm{l}$ of whole blood samples. Pipettes were shaken repeatedly for mixing of the whole blood samples. The blood cells were lysed for 5-10 min at room temperature until fully disrupted and then stored in a refrigerator at $-80^{\circ} \mathrm{C}$. Total RNA was extracted as soon as possible.

RNA extraction. Total RNA containing small RNA was extracted from blood using the TRI LS pure reagent and was purified with Qiagen miRNeasy Mini kit (Qiagen GmbH, Hilden, Germany) according to the manufacturer's instructions. The purity and concentration of RNA were determined by $\mathrm{OD}_{260} / \mathrm{OD}_{280}$ readings using a NanoDrop ND-1000 spectrophotometer (NanoDrop Technologies; Thermo Fisher Scientific, Inc., Wilmington, DE, USA). RNA integrity was determined using $1 \%$ formaldehyde denaturing gel electrophoresis. The extracted RNAs were digested, dephosphorylated, denatured, amplified, and labeled with Cy3-dCTP according to the manufacturer's instructions (11). The purified RNAs were hybridized to a microarray (CircRNA Array v2; CapitalBio, Corp.) containing 170,340 human circRNA probes and a microarray (Agilent Human miRNA Microarray, release 21.0, $8 \times 60 \mathrm{~K}$; Agilent Technologies, Inc.) containing 2,568 human miRNA probes.

Microarray imaging and data analysis. The microarray data of the circRNA and miRNA array were analyzed for data summarization, normalization, and quality control using GeneSpring software v13.0 (Agilent Technologies, 
Table II. Basic information of inpatients for the expression profiles of circRNA and miRNA.

\begin{tabular}{|c|c|c|}
\hline Variables & Control group & Test group \\
\hline Age (years) & $51.6 \pm 10.44$ & $61.25 \pm 9.38$ \\
\hline \multicolumn{3}{|l|}{ Sex } \\
\hline Male & 5 & 12 \\
\hline Female & 15 & 8 \\
\hline HR (beats/min) & $68.6 \pm 15.8$ & $69.95 \pm 20.16$ \\
\hline $\mathrm{BP}(\mathrm{mmHg})$ & $138.30 \pm 15.06$ & $137.00 \pm 17.08$ \\
\hline FBG (mmol/l) & $6.17 \pm 2.87$ & $6.21 \pm 2.23$ \\
\hline $\mathrm{CHO}(\mathrm{mmol} / \mathrm{l})$ & $4.18 \pm 0.80$ & $4.58 \pm 1.18$ \\
\hline TG (mmol/l) & $1.81 \pm 1.30$ & $1.79 \pm 0.91$ \\
\hline APOA1 (g/l) & $1.18 \pm 0.18$ & $1.09 \pm 0.24$ \\
\hline APOB (g/l) & $1.02 \pm 0.80$ & $0.89 \pm 0.24$ \\
\hline HDL (mmol/l) & $1.16 \pm 0.20$ & $1.09 \pm 0.35$ \\
\hline $\mathrm{LDL}(\mathrm{mmol} / \mathrm{l})$ & $2.53 \pm 0.91$ & $2.72 \pm 0.89$ \\
\hline $\operatorname{Lp}(\mathrm{a})(\mathrm{g} / \mathrm{l})$ & $0.267 \pm 0.25$ & $0.40 \pm 0.39$ \\
\hline AST (U/1) & $31.35 \pm 34.34$ & $27.75 \pm 15.04$ \\
\hline LDH (U/l) & $67.60 \pm 60.32$ & $66.80 \pm 61.40$ \\
\hline HBDH (U/l) & $144.30 \pm 30.44$ & $177.95 \pm 129.54$ \\
\hline CK (U/1) & $67.35 \pm 22.02$ & $103.40 \pm 67.54$ \\
\hline CK-MB (U/l) & $13.70 \pm 6.43$ & $14.40 \pm 6.18$ \\
\hline $\mathrm{EF}$ & $63.47 \pm 3.68$ & $60.42 \pm 3.68$ \\
\hline FS & $36.23 \pm 7.14$ & $32.47 \pm 5.72$ \\
\hline
\end{tabular}

circRNA, circular RNA; miRNA, microRNA; HR, heart rate; BP, blood pressure; FBG, fasting blood glucose; $\mathrm{CHO}$, total cholesterol; TG, triglycerides; APOA1, apolipoprotein A1; APOB, apolipoprotein B; HDL, high-density lipoprotein; LDL, low-density lipoprotein; Lp(a), lipoprotein (a); AST, aspartate aminotransferase; LDH, lactate dehydrogenase; $\mathrm{HBDH}$, hydroxybutyrate dehydrogenase; $\mathrm{CK}$, creatine kinase; CK-MB, creatine kinase-MB EF, ejection fraction; FS, fractional shortening.

Inc.). To select the differentially expressed genes, we used threshold values of $\geq 2$ (miRNA $\geq 1$ ) fold change and a t-test $\mathrm{P}<0.05$. The data was $\log 2$ transformed and median centered by genes using the adjust data function in Cluster 3.0 software (Cluster Software, Inc., Columbus, OH, USA). Then they were further analyzed with hierarchical clustering with average linkage (12). The genes were differentially expressed in the two groups. The selected circRNAs were then verified by quantitative polymerase chain reaction (qPCR), which contained 15 control subjects and 15 patients with different severities of CHD, and then the microarray tool was used for pathway and Gene Ontology (GO) term analysis to evaluate the differentially expressed circRNAs and any corresponding miRNAs. Finally, we performed tree visualizations using Java TreeView (Stanford University School of Medicine, Stanford, CA, USA).

Construction of the circRNA-miRNA network. circRNAmiRNA network was constructed based on miRanda v3.3

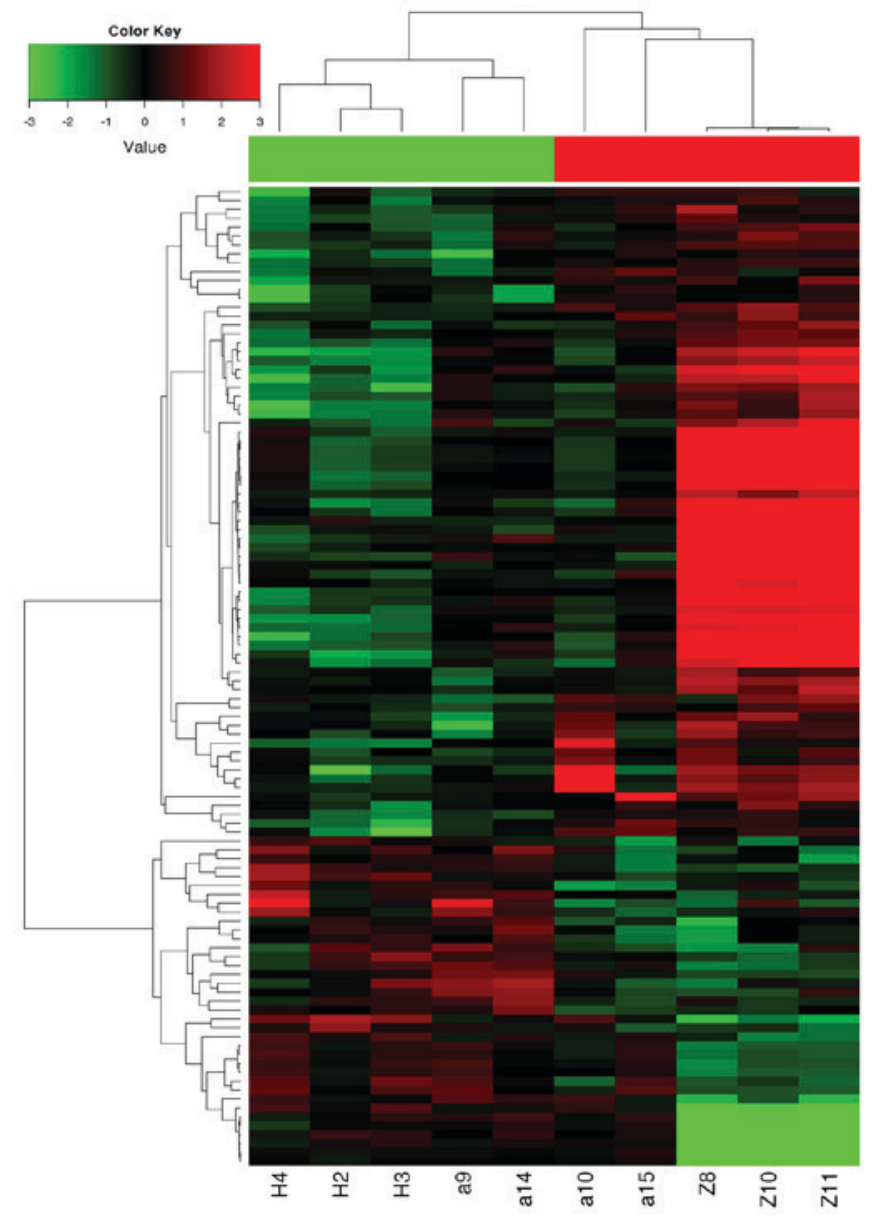

Figure 1. Map of cluster analysis for CHD. The heat map is constructed by hierarchical cluster analysis. Red and green indicate up- and downregulation during circRNA, respectively. The heat map includes 110 gene expression levels which were significantly altered by CHD. The genes in 10 clusters, denoted as group $1(\mathrm{a} 9, \mathrm{a} 14, \mathrm{H} 2, \mathrm{H} 3, \mathrm{H} 4)$ and group 2 (a10, a15, Z8, Z10, Z11), were 73 upregulated and 37 downregulated. CHD, coronary heart disease; circRNA, circular RNA.

software combined with entropy values $<20$. These circRNA-miRNA pairs were chosen to construct the network using the open source bioinformatics software Cytoscape. In a network analysis, the degree centrality is defined as the number of links between one node and the others. A degree is the simplest and most important measure of a gene centrality within a network determining the relative importance (13).

\section{Results}

Research subjects. The data of the present research were collected from a total of 40 inpatients admitted to the First Affiliated Hospital of Xinxiang Medical University, between November 2016 to February 2017, with CHD and GS<3 (control group) or GS >40 (test group).

General information. General information of patients were collected including coronary GS (Table I), age, sex, BP, FBG, CHO, TG, HDL, LDL, EF and FS (Table II). Standard values: FBG, 3.9-6.1 mmol; CHO, 0-5.2 mmol; TG, 0.7-1.7 mmol; APOA1, 1-1.76 g/l; APOB, 0.6-1.14 g/l; HDL, 0.8-1.55 mmol/1; LDL, 1.64-3.62 mmol/1; Lp(a), 0-0.3 g/l. 
Table III. Expression profiles of circRNAs that were upregulated $(\mathrm{P}<0.05, \mathrm{FC} \geq 2)$.

\begin{tabular}{|c|c|c|c|c|c|c|c|c|c|}
\hline No. & Probe name & P-value & $\mathrm{FC}(\mathrm{abs})$ & Gene symbol & Regulation & Chromosome & Strand & Start & End \\
\hline 1 & hsa_circ_0140759 & 0.031 & 45.35329 & UTY & Up & $\operatorname{chrY}$ & - & 15466882 & 15481229 \\
\hline 2 & hsa_circ_16316-13 & 0.046 & 28.26892 & UTY & Up & $\operatorname{chrY}$ & - & 15447442 & 15478273 \\
\hline 3 & hsa_circ_0140760 & 0.045 & 28.18893 & UTY & Up & $\operatorname{chrY}$ & - & 15467172 & 15471765 \\
\hline 4 & hsa_circ_0140758 & 0.047 & 25.23608 & UTY & Up & $\operatorname{chrY}$ & - & 15447442 & 15448215 \\
\hline 5 & hsa_circ_16316-11 & 0.039 & 16.33733 & UTY & Up & $\operatorname{chrY}$ & - & 15471646 & 15471866 \\
\hline 6 & hsa_circ_0140781 & 0.040 & 12.63422 & KDM5D & Up & $\operatorname{chrY}$ & - & 21901413 & 21903743 \\
\hline 7 & hsa_circ_0140736 & 0.043 & 12.46352 & USP9Y & Up & $\operatorname{chrY}$ & + & 14821320 & 14885859 \\
\hline 8 & hsa_circ_16316-9 & 0.026 & 10.90928 & UTY & Up & $\operatorname{chrY}$ & - & 15435434 & 15438230 \\
\hline 9 & hsa_circ_0140746 & 0.033 & 10.40101 & USP9Y & Up & $\operatorname{chrY}$ & + & 14870435 & 14885859 \\
\hline 10 & hsa_circ_0009024 & 0.020 & 9.696754 & - & Up & $\operatorname{chrY}$ & + & 21749095 & 21749393 \\
\hline 11 & hsa_circ_16316-12 & 0.017 & 9.500227 & UTY & Up & $\operatorname{chrY}$ & - & 15435434 & 15448215 \\
\hline 12 & hsa_circ_0007907 & 0.024 & 8.476297 & ZFY & Up & chrY & + & 2829114 & 2829687 \\
\hline 13 & hsa_circ_0140732 & 0.031 & 7.912563 & USP9Y & Up & chrY & + & 14813938 & 14834120 \\
\hline$\cdots$ & $\cdots \cdots$ & & & & & & & & \\
\hline 73 & hsa_circ_0099619 & 0.003 & 2.00747 & NEDD1 & Up & $\operatorname{chr} 12$ & + & 97264281 & 97303673 \\
\hline
\end{tabular}

Dotted line indicates data not shown. circRNA, circular RNA.

Table IV. Expression profiles of circRNAs that were downregulated $(\mathrm{P}<0.05, \mathrm{FC} \geq 2)$.

\begin{tabular}{|c|c|c|c|c|c|c|c|c|c|}
\hline No. & Probe name & P-value & $\mathrm{FC}$ (abs) & Gene symbol & Regulation & Chromosome & Strand & Start & End \\
\hline 1 & hsa_circ_0091074 & 0.039 & 20.66278 & - & Down & $\operatorname{chrX}$ & - & 73048902 & 73051109 \\
\hline 2 & hsa_circ_0140538 & 0.037 & 15.17814 & - & Down & $\operatorname{chrX}$ & - & 73045949 & 73051109 \\
\hline 3 & hsa_circ_0091073 & 0.044 & 14.23409 & - & Down & $\operatorname{chrX}$ & - & 73040494 & 73051109 \\
\hline 4 & hsa_circ_0140539 & 0.046 & 11.15955 & - & Down & $\operatorname{chrX}$ & - & 73045949 & 73057338 \\
\hline 5 & hsa_circ_16166-3 & 0.046 & 8.908921 & - & Down & $\operatorname{chrX}$ & - & 73050900 & 73053209 \\
\hline 6 & hsa_circ_16166-1 & 0.049 & 8.020764 & - & Down & $\operatorname{chrX}$ & - & 73050900 & 73057338 \\
\hline 7 & hsa_circ_0140536 & 0.034 & 5.790904 & ABCA5 & Down & $\operatorname{chr} X$ & - & 73044087 & 73044570 \\
\hline 8 & hsa_circ_0107597 & 0.028 & 4.049471 & - & Down & chr17 & - & 67270099 & 67305564 \\
\hline 9 & hsa_circ_0140541 & 0.031 & 3.919625 & SCN9A & Down & $\operatorname{chr} \mathrm{X}$ & - & 73046801 & 73046954 \\
\hline 10 & hsa_circ_0117953 & 0.002 & 2.986698 & CPNE8 & Down & chr2 & - & $1.67 \times 10^{8}$ & $1.67 \times 10^{8}$ \\
\hline 11 & hsa_circ_0098463 & 0.048 & 2.7141 & DNAH14 & Down & chr12 & - & 39117573 & 39156018 \\
\hline 12 & hsa_circ_770-66 & 0.042 & 2.692528 & VWA8 & Down & chr1 & + & $2.25 \times 10^{8}$ & $2.25 \times 10^{8}$ \\
\hline 13 & hsa_circ_0030090 & 0.045 & 2.620312 & GPSM2 & Down & chr13 & - & 42259182 & 42335340 \\
\hline & $\cdots \cdots$ & $\cdots \cdots$ & & & & & & & \\
\hline 37 & hsa_circ_0079838 & 0.030 & 2.000309 & DPY19L1 & Down & chr7 & - & 34989408 & 35013217 \\
\hline
\end{tabular}

Dotted line indicates data not shown. circRNA, circular RNA.

Expression profiles of circRNA and miRNA in the peripheral blood of CHD patients. The mechanism of CHD was investigated with high-throughput technology in the peripheral blood of CHD patients.

Expression profiles of circRNAs. There was a significant difference between test and control group. The results showed clear differences in the expression profiles of circRNAs between test and control groups (Fig. 1). Differential expression was detected in a total of 110 circRNAs, of which 73 were upregulated and 37 were downregulated in the CHD group $(\mathrm{P}<0.05, \mathrm{FC} \geq 2)$. circRNAs were found to be related to UTY, KDM5D, USP9Y, ABCA5, SCN9A, CPNE8, and other genes (Tables III and IV). Among the 110 circRNAs, we verified 6 candidate biomarkers by qPCR, and 6 circRNAs with the highest fold changes $(\mathrm{P}<0.01)$, which were taken for further analysis: hsa_circ_0030769, hsa_circ_0079828, hsa_ circ_15486-161, hsa_circ_0122274, hsa_circ_16316-13 and hsa_circ_0140538. The results confirmed the findings of the microarray analysis, as the levels of these hsa_circ_16316-13 were significantly increased in the CHD patients. 
Table V. Expression profiles of miRNAs $(\mathrm{P}<0.05, \mathrm{FC} \geq 1)$.

\begin{tabular}{|c|c|c|c|c|}
\hline No. & Systematic name & P-value & $\mathrm{FC}(\mathrm{abs})$ & Regulation \\
\hline 1 & hsa-miR-148a-3p & 0.001001 & 1.411098 & Up \\
\hline 2 & hsa-miR-1260a & 0.007008 & 1.454044 & Up \\
\hline 3 & hsa-miR-29c-3p & 0.008573 & 1.333568 & Up \\
\hline 4 & hsa-miR-101-5p & 0.015351 & 1.268211 & Up \\
\hline 5 & hsa-miR-545-3p & 0.020073 & 1.175692 & Up \\
\hline 6 & hsa-miR-5100 & 0.020456 & 2.086728 & Up \\
\hline 7 & hsa-miR-194-5p & 0.023195 & 1.715863 & Up \\
\hline 8 & hsa-miR-215-5p & 0.02325 & 1.404127 & Up \\
\hline 9 & hsa-miR-4443 & 0.035173 & 1.384234 & Up \\
\hline 10 & hsa-miR-19a-3p & 0.039729 & 1.331585 & Up \\
\hline 11 & hsa-miR-32-5p & 0.042593 & 1.549326 & Up \\
\hline 12 & hsa-miR-103a-3p & 0.043258 & 1.156287 & Up \\
\hline 13 & hsa-miR-424-5p & 0.044219 & 1.279992 & Up \\
\hline 14 & hsa-miR-3196 & 0.009454 & 1.148938 & Down \\
\hline 15 & hsa-miR-3651 & 0.010955 & 1.485764 & Down \\
\hline 16 & hsa-miR-1249-3p & 0.037988 & 1.325854 & Down \\
\hline 17 & hsa-miR-4281 & 0.042548 & 1.095865 & Down \\
\hline 18 & hsa-miR-1228-3p & 0.042683 & 1.518054 & Down \\
\hline
\end{tabular}

miRNA, microRNA.

Table VI. Coexpression of circRNA-miRNA $(\mathrm{P}<0.05, \mathrm{FC}>2)$.

\begin{tabular}{|c|c|c|c|c|c|c|c|c|}
\hline No. & Probe name & $\begin{array}{l}\text { miRNA capable } \\
\text { of bonding } \\
\text { to } \geq 1 \text { circRNA }\end{array}$ & $\begin{array}{l}\text { miRNA capable } \\
\text { of bonding } \\
\text { to } \geq 2 \text { circRNA }\end{array}$ & Regulation & $\begin{array}{c}\text { Gene } \\
\text { symbol }\end{array}$ & Strand & Start & End \\
\hline 1 & hsa_circ_16316-13 & 100 & 100 & Up & UTY & - & 15447442 & 15478273 \\
\hline 2 & hsa_circ_0140760 & 100 & 100 & Up & UTY & - & 15467172 & 15471765 \\
\hline 3 & hsa_circ_0140758 & 100 & 100 & Up & UTY & - & 15447442 & 15448215 \\
\hline 4 & hsa_circ_0140736 & 100 & 100 & Up & USP9Y & + & 14821320 & 14885859 \\
\hline 5 & hsa_circ_16316-12 & 100 & 100 & Up & UTY & - & 15435434 & 15448215 \\
\hline 6 & hsa_circ_0140732 & 100 & 100 & Up & USP9Y & + & 14813938 & 14834120 \\
\hline 7 & hsa_circ_0140783 & 100 & 100 & Up & - & - & 22669237 & 22683186 \\
\hline 8 & hsa_circ_0140757 & 100 & 100 & Up & UTY & - & 15435434 & 15472408 \\
\hline 9 & hsa_circ_16316-14 & 100 & 100 & Up & UTY & - & 15435434 & 15478273 \\
\hline 10 & hsa_circ_0140756 & 100 & 100 & Up & UTY & - & 15409586 & 15438230 \\
\hline 11 & hsa_circ_0140733 & 100 & 100 & Up & USP9Y & + & 14813938 & 14870572 \\
\hline 12 & hsa_circ_0140779 & 100 & 100 & Up & - & + & 21749095 & 21752658 \\
\hline 13 & hsa_circ_0092240 & 100 & 100 & Up & USP9Y & + & 14832521 & 14905134 \\
\hline 14 & hsa_circ_0092257 & 100 & 83 & Up & USP9Y & + & 14954988 & 14969586 \\
\hline$\cdots$ & $\ldots \ldots$ & $\ldots \ldots$ & $\ldots \ldots$ & & & & & \\
\hline 73 & hsa_circ_8216-2 & 1 & 0 & Up & $\mathrm{ZFY}$ & + & 2821949 & 2822038 \\
\hline
\end{tabular}

Dotted line indicates data not shown. circRNA, circular RNA; miRNA, microRNA.

Expression profiles of miRNAs. The results showed significant differences in the expression profiles of miRNAs between test and control group. Differential expression was detected in a total of 18 miRNAs $(\mathrm{P}<0.05, \mathrm{FC} \geq 1)$, of which
13 were upregulated and 5 were downregulated in the CHD group (Table V). We verified 2 candidate miRNAs by qPCR: hsa-let-7c-5p and hsa-miR-101-5p were significantly expressed in the CHD patients. 
Table VII. Number of coexpressed circRNA-miRNA ( $\mathrm{P}<0.05, \mathrm{FC}>2$-fold).

\begin{tabular}{|c|c|c|c|c|c|c|c|c|}
\hline No. & Probe name & $\begin{array}{l}\text { miRNA capable } \\
\text { of bonding } \\
\text { to } \geq 1 \text { circRNA }\end{array}$ & $\begin{array}{l}\text { miRNA capable } \\
\text { of bonding to } \\
\text { to } \geq 2 \text { circRNA }\end{array}$ & Regulation & $\begin{array}{l}\text { Gene } \\
\text { symbol }\end{array}$ & Strand & Start & End \\
\hline 1 & hsa_circ_0140538 & 100 & 100 & Down & - & - & 73045949 & 73051109 \\
\hline 2 & hsa_circ_0091073 & 100 & 100 & Down & - & - & 73040494 & 73051109 \\
\hline 3 & hsa_circ_0140539 & 100 & 100 & Down & - & - & 73045949 & 73057338 \\
\hline 4 & hsa_circ_0107597 & 100 & 6 & Down & ABCA5 & - & 67270099 & 67305564 \\
\hline 5 & hsa_circ_0030090 & 100 & 7 & Down & VWA8 & - & 42259182 & 42335340 \\
\hline 6 & hsa_circ_0122274 & 100 & 11 & Down & ATR & - & $1.42 \times 10^{8}$ & $1.42 \times 10^{8}$ \\
\hline 7 & hsa_circ_15486-161 & 100 & 23 & Down & PRKDC & - & 48817428 & 48867006 \\
\hline 8 & hsa_circ_0015545 & 100 & 100 & Down & GLUL & - & $1.82 \times 10^{8}$ & $1.82 \times 10^{8}$ \\
\hline 9 & hsa_circ_0105530 & 100 & 4 & Down & CHD9 & + & 53189837 & 53243725 \\
\hline 10 & hsa_circ_0091221 & 100 & 20 & Down & CSTF2 & + & $1 \times 10^{8}$ & $1 \times 10^{8}$ \\
\hline 11 & hsa_circ_0041763 & 100 & 75 & Down & ACADVL & + & 7123782 & 7128585 \\
\hline 12 & hsa_circ_0134130 & 100 & 3 & Down & - & - & 32625588 & 32678977 \\
\hline 13 & hsa_circ_0064254 & 100 & 49 & Down & FANCD2 & + & 10119764 & 10143614 \\
\hline 14 & hsa_circ_0091075 & 89 & 5 & Down & - & + & 73164418 & 73167209 \\
\hline ...... & & $\ldots$ & $\ldots$ & Down & & & & \\
\hline 37 & hsa_circ_0093369 & 4 & 0 & Down & THNSL1 & - & 25313675 & 25313802 \\
\hline
\end{tabular}

Dotted line indicates data not shown. circRNA, circular RNA; miRNA, microRNA.

Table VIII. Top 8 circRNAs-miRNA.

\begin{tabular}{llccc}
\hline No. & Probe name & P-value & miRNA no. & Regulation \\
\hline 1 & hsa_circ_0140759 & 0.031 & 6 & $\mathrm{Up}$ \\
2 & hsa_circ_16316-13 & 0.046 & 17 & $\mathrm{Up}$ \\
3 & hsa_circ_0140760 & 0.045 & 66 & $\mathrm{Up}$ \\
4 & hsa_circ_0140758 & 0.047 & 3 & $\mathrm{Up}$ \\
5 & hsa_circ_0140736 & 0.043 & 7 & $\mathrm{Up}$ \\
6 & hsa_circ_0140781 & 0.040 & 1 & $\mathrm{Up}$ \\
7 & hsa_circ_0140538 & 0.036 & 100 & Down \\
8 & hsa_circ_0091073 & 0.044 & 100 & Down \\
\hline
\end{tabular}

circRNA, circular RNA; miRNA, microRNA.

circRNA cluster analysis. Cluster analysis was performed on the circRNA between test and control group. Because there were not sufficient data concerning miRNA, no cluster analysis was performed as shown in Fig. 1.

Coexpression of circRNA-miRNA. Examination of the coexpression of circRNA-miRNA to the GS of CHD showed that a large number of circRNAs take part in the CHD process and may influence a similarly large number of miRNAs, and circRNAs participate in the regulation of $>100$ miRNAs, such as upregulated hsa_circ_16316-13, hsa_circ_0140760, hsa_circ_0140748; and downregulated hsa_circ_0140538, hsa_circ_0091073, hsa_circ_0140539 (Tables VI-VIII, Fig. 2).

Enrichment analysis. Enrichment analysis of the data from the expression profiles of circRNAs $(\mathrm{P}<0.05, \mathrm{FC} \geq 2)$ indicated

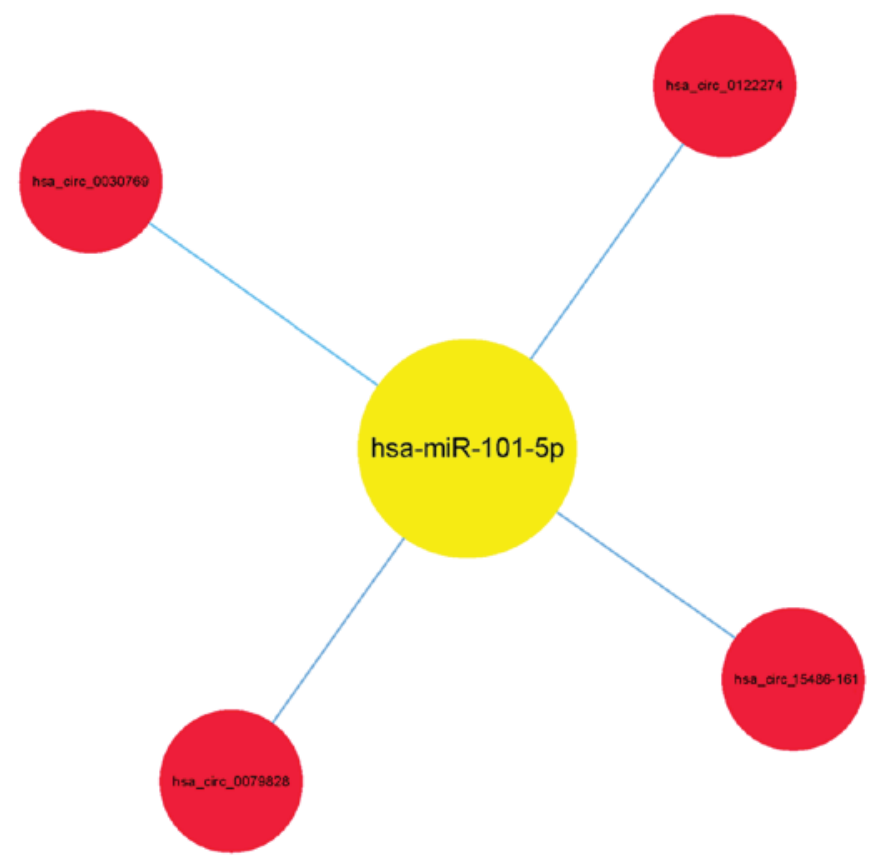

Figure 2. Map of coexpression of circRNA-miRNA for CHD. In the map hsa-miR-101-5p is associated with hsa_circ_0030769, hsa_circ_15486-161, hsa_circ_0122274, and hsa_circ_0079828. circRNA, circular RNA; miRNA, microRNA; CHD, coronary heart disease.

statistically significant difference between test and control group using NHGRI GWAS Catalog, GO, and Reactome.

Disease enrichment analysis. The analysis of disease enrichment was performed using the NHGRI GWAS Catalog. The results showed that circRNA is involved in a 
Significant Enriched NHGRI_GWAS_Catalog.disease Terms (Top 30)

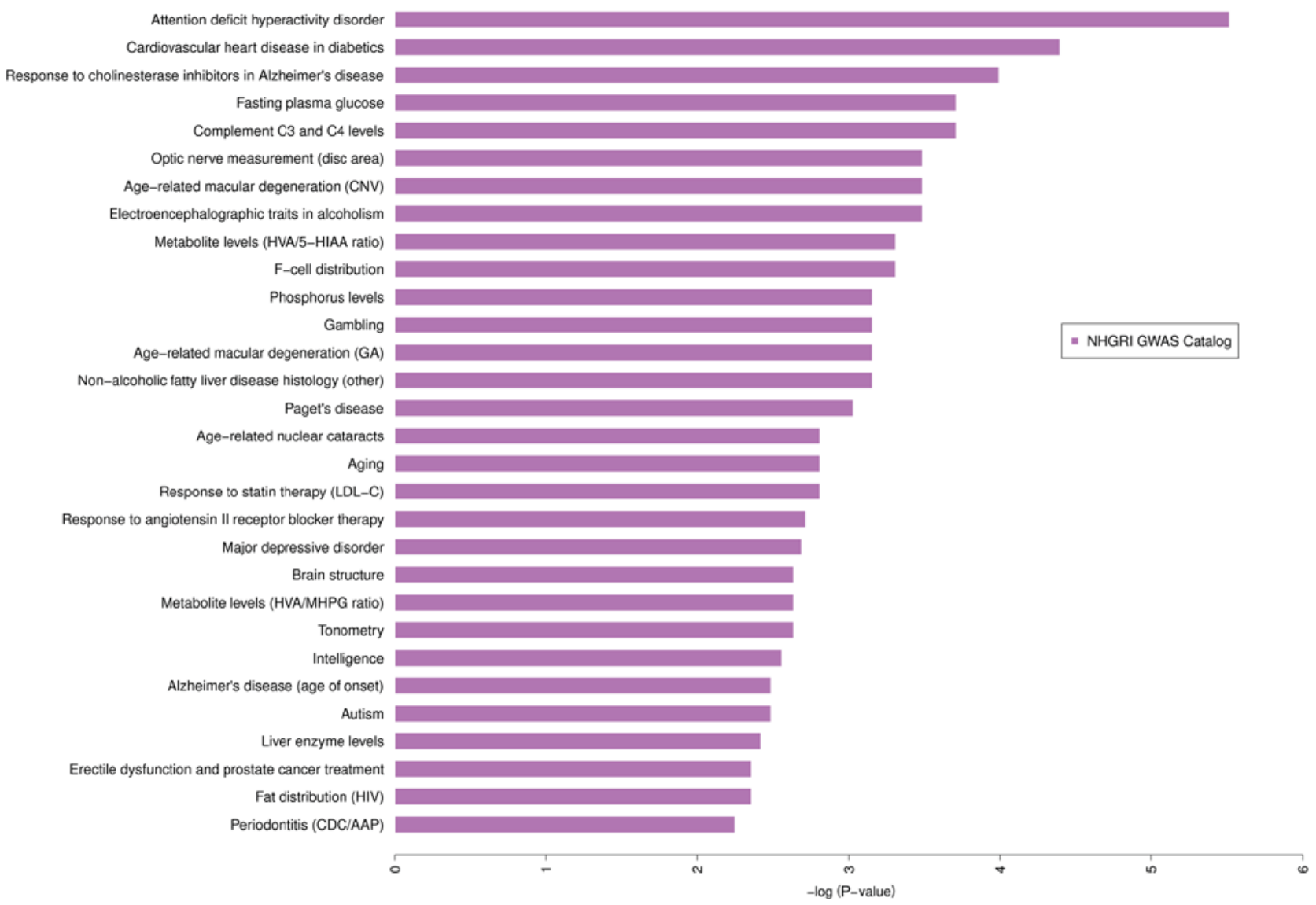

Figure 3. Significantly enriched NHGRI_GWAS_Catalog disease terms for CHD. The significantly enriched NHGRI_GWAS_Catalog disease terms include: attention deficit hyperactivity disorder, cardiovascular heart disease in diabetics, response to cholinesterase inhibitors in Alzheimer's disease, complement C3 and C4 levels, fasting plasma glucose, electroencephalographic traits in alcoholism, age-related macular degeneration, optic nerve measurement (disc area), F-cell distribution, metabolite levels (HVA/5-HIAA ratio), non-alcoholic fatty liver disease histology (other), age-related macular degeneration (GA), gambling, phosphorus levels, Paget's disease. CHD, coronary heart disease.

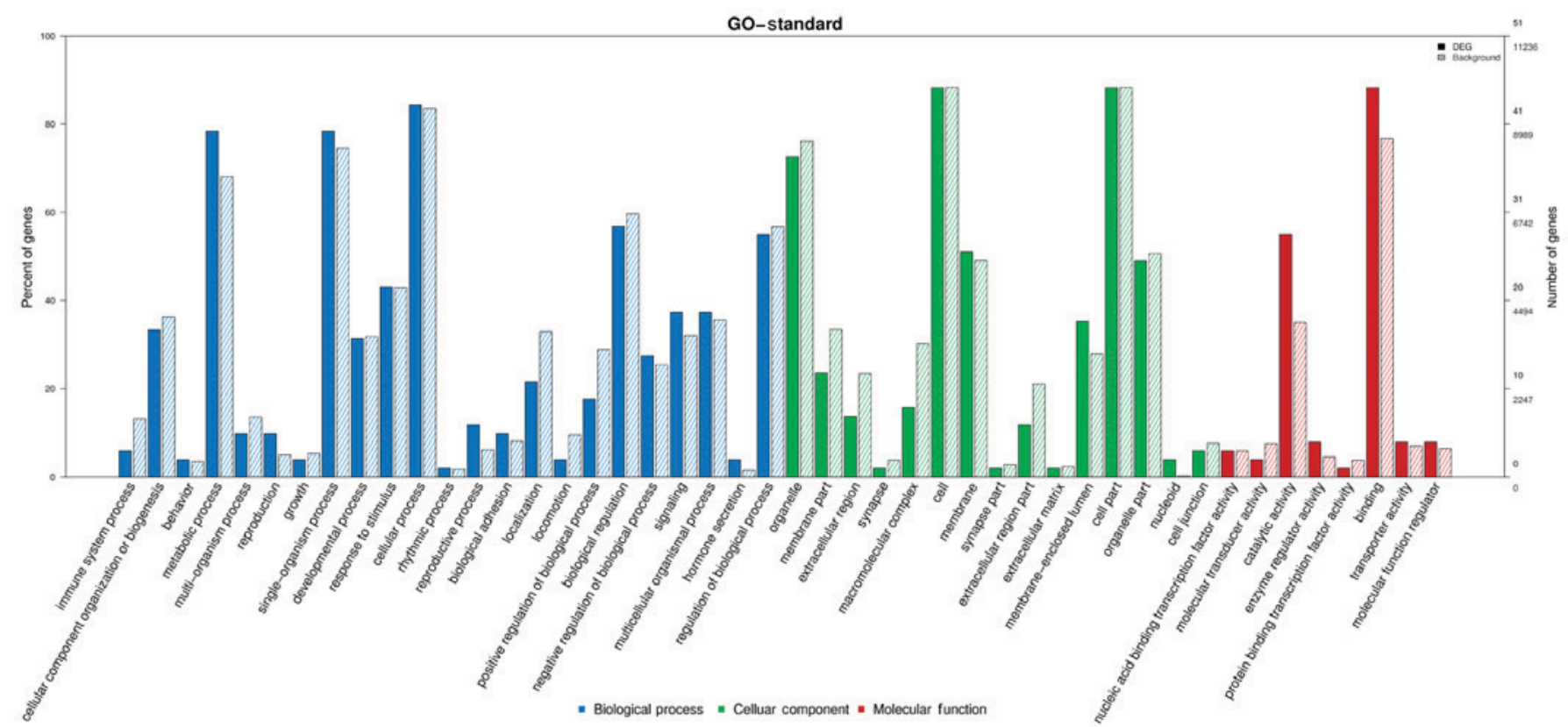

Figure 4. GO enrichment for CHD. Enrichment analysis is a technique commonly used to interpret a list of genes (such as the list of significant genes in Tables III and IV). GO for CHD is divided into biological processes, cellular components and molecular functions. Metabolic process, single-organism process, cellular process, biological regulation, and regulation of biological process are significantly high in the biological process category. Organelle, cell, and cell part are significantly high in the cellular component category. Catalytic activity and binding are significantly high in the molecular function category. GO, Gene Ontology; CHD, coronary heart disease. 


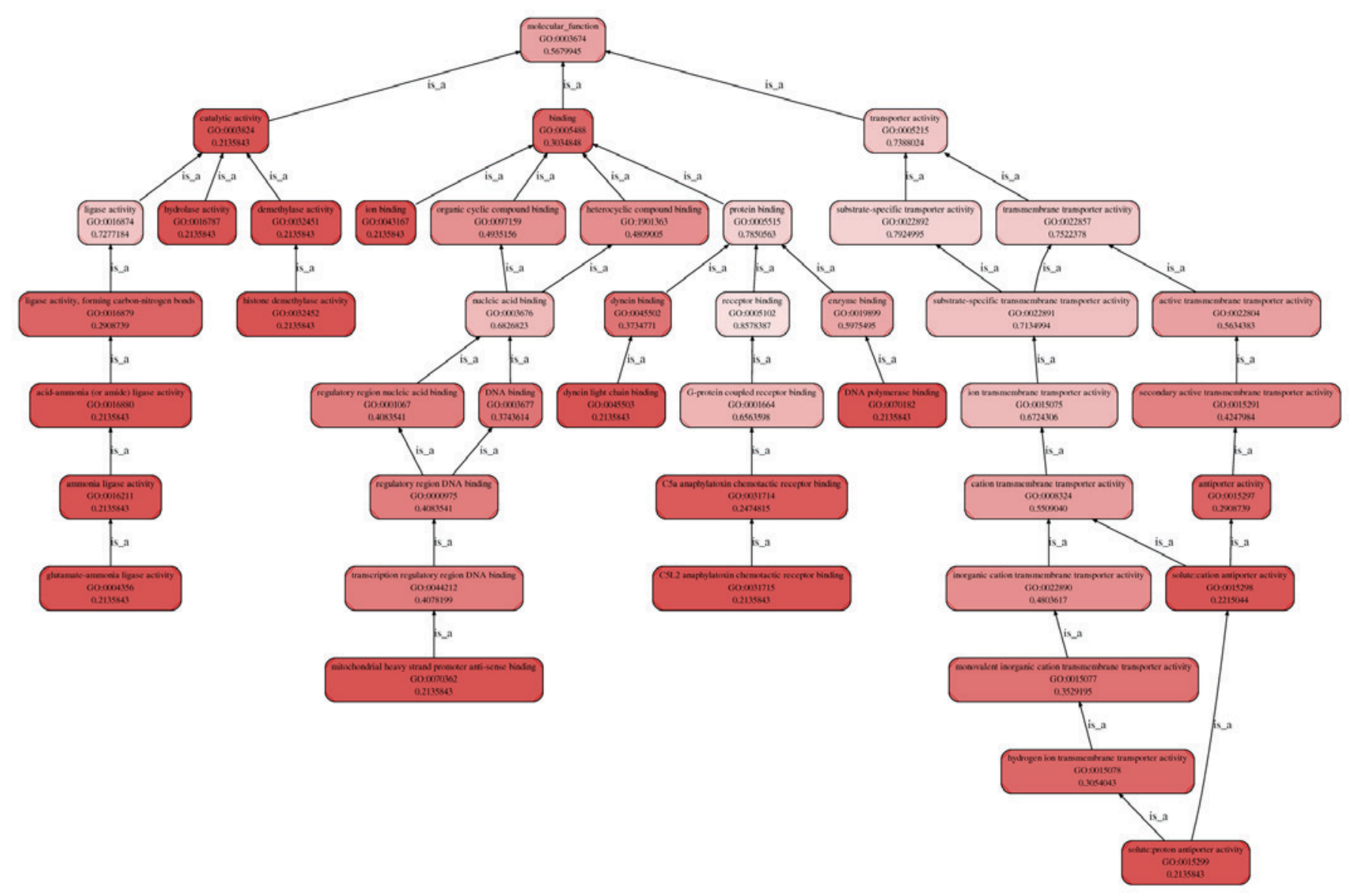

Figure 5. Enrichment.Go.hierarchy.molecular_function for CHD. The top 10 molecular functions are: DNA polymerase binding, ion binding, solute:proton antiporter activity, hydrolase activity, catalytic activity, histone demethylase activity, demethylase activity, dynein light chain binding, mitochondrial heavy strand promoter anti-sense binding, glutamate-ammonia ligase activity. The molecular function category includes four fields: the glutamate-ammonia ligase activity, mitochondrial heavy strand promoter anti-sense binding, C5L2 anaphylatoxin chemotactic receptor binding, and solute:proton antiporter activity. These influence the overall catalytic, binding, and transporter activity, finally changing the molecular function. CHD, coronary heart disease.

total of 59 diseases and significantly involved in 15 diseases ( $\mathrm{P}<0.05$; Fig. 3).

$G O$ enrichment analysis. $G O$ is divided into biological processes, cellular components, and molecular functions (Figs. 4 and 5). i) The biological processes associated with circRNA tended to relate more to content, and the results showed circRNA to be involved in a total of 1,254 terms and significantly involved in 136 biological processes $(\mathrm{P}<0.05)$. ii) Results showed circRNA to be involved in a total of 269 terms and significantly involved in 89 molecular functions $(\mathrm{P}<0.05)$. iii) The cellular component category contains a total of 171 terms, 21 of which were found to be significant $(\mathrm{P}<0.05)$.

Reactome pathway. By analyzing data of 110 statistical significant differences $(\mathrm{P}<0.05, \mathrm{FC} \geq 2)$ on the Reactome pathway analysis of circRNAs from the experimental and control group, we found that it has 172 pathways significantly involved in $30(\mathrm{P}<0.05$; Fig. 6$)$.

\section{Discussion}

CHD still represents the leading cause of mortality and morbidity worldwide, and the underlying disease, atherosclerosis, as a common feature of the CHD, is initiated and propagated by continuous damage, as a result of a variety of factors. In the regulation of health and disease, circRNAs act as urgent effectors by miRNA sponges, splicing or transcriptional regulators, and agents interacting with RBPs.

Long non-coding RNA (IncRNA) works and controls the atherosclerosis process (14). There is growing evidence that non-coding RNA (circRNA and miRNA) is involved in the development and progression of CHD. The association of circRNA with CHD began with the study of antisense non-coding RNA in the INK4 locus, ANRIL. In 2010, Burd et al found that circANRIL expression by human INK4/ARF transcriptional regulation is associated the atherosclerotic risk (15). The same year, it was confirmed that ANRIL expression is associated with atherosclerosis risk at chromosome 9p21 (16). In 2016, Holdt et al found circANRIL, a prototype of a circRNA regulating ribosome biogenesis and conferring atheroprotection, and suggested that circANRIL remains a potential therapeutic target for the treatment of atherosclerosis (17). Studies have illustrated that circRNA can contribute to atherosclerosis development and progression. In addition, circRNA hsa_circ_0124644 can be used for the diagnosis of coronary artery disease (7). A circRNA, termed heart-related circRNA (HRCR), has been shown to act as an endogenous miR-223 sponge to inhibit cardiac hypertrophy and heart failure (18). circRNAs are dynamically expressed in a human induced pluripotent stem cell-derived cardiomyocytes model of cardiac development 


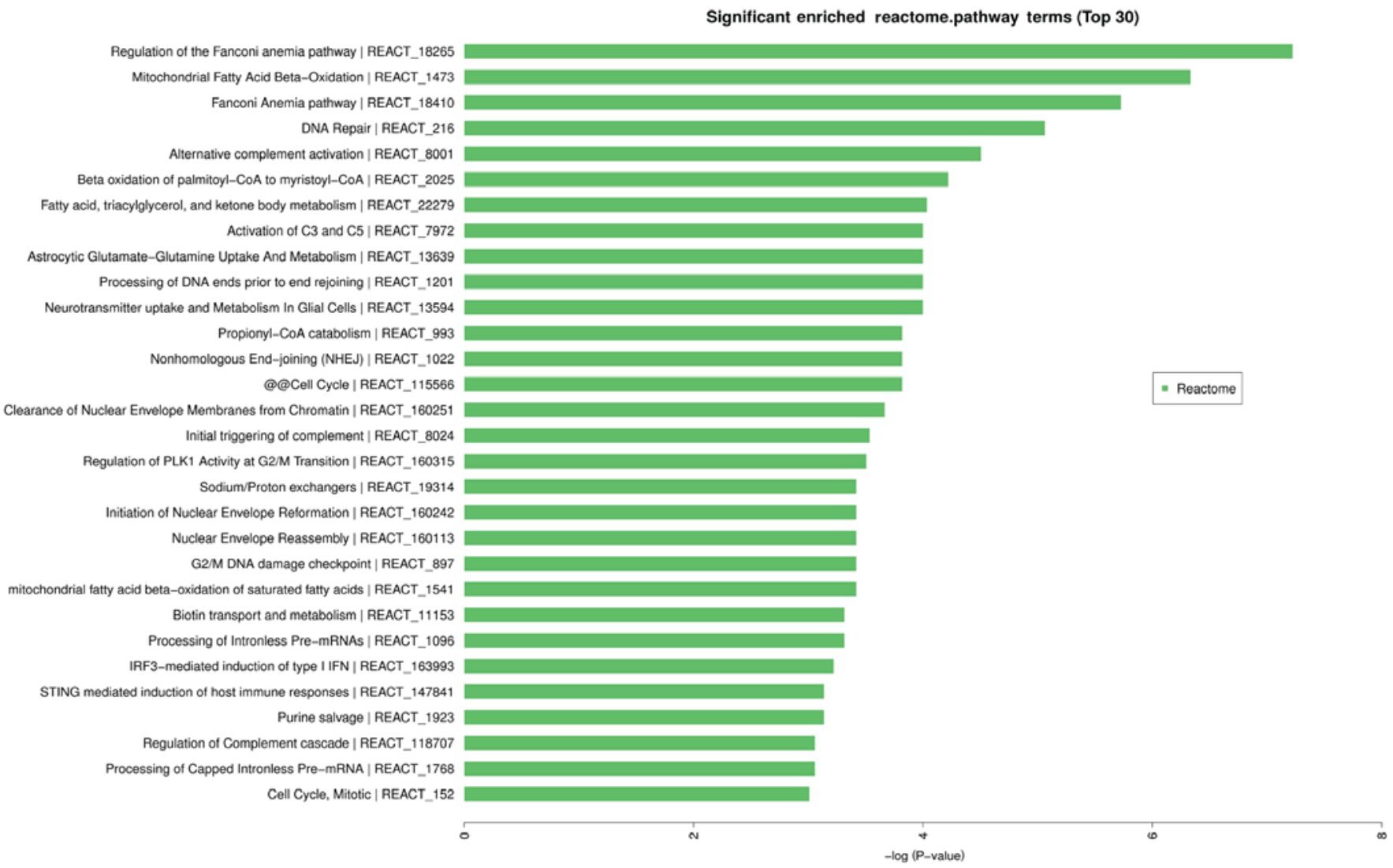

Figure 6. Enriched Reactome pathway terms for CHD. The top 10 Reactome pathway terms are: regulation of the Fanconi anemia pathway, mitochondrial fatty acid $\beta$-oxidation, Fanconi anemia pathway, DNA repair, alternative complement activation, $\beta$ oxidation of palmitoyl-CoA to myristoyl-CoA, fatty acid triacylglycerol and ketone body metabolism, neurotransmitter uptake and metabolism in glial cells, processing of DNA ends prior to end rejoining, astrocytic glutamate-glutamine uptake and metabolism. CHD, coronary heart disease.

and stress response. circRNAs such as circSLC8A1, circCACNA1D, circSPHKAP and circALPK2 may serve as biomarkers of cardiomyocytes (CMs), and circSLC8A1 is increased abnormally in heart tissues of patients with dilated cardiomyopathy $(19,20)$. Through microarray analysis, the results of this study revealed that the mechanism of CHD may be related to the expression of circRNA and miRNA and the co-expression of circRNA-miRNA, as well as circ_0091074, hsa_circ_0140538, and hsa_circ_0140539 may act on hsa-miR-101-5p, hsa-miR-148a-3p, and hsa-miR-1260a and may be involved in CHD.

The results of enrichment analysis of the data from the expression profiles of circRNAs indicated statistically significant difference between test and control group using NHGRI GWAS Catalog, GO, and Reactome. For example, cardiovascular heart disease in diabetics is included in the result analysis of disease enrichment. The result of enrichment analysis of GO showed that circRNA may be additionally involved in other than the biological process, molecular function and cellular component. It is important that the expression profile changes of circRNA are involved in lipid metabolism pathway, for example, mitochondrial fatty acid $\beta$-oxidation, $\beta$ oxidation of palmitoyl-CoA to myristoyl-CoA, fatty acid-triacylglycerol-and ketone body metabolism, mitochondrial fatty acid $\beta$-oxidation of saturated fatty acids.

The mechanisms involved in cardiovascular aging, and the potential for targeting novel pathways implicated in endothelial dysfunction, mitochondrial oxidative stress, chromatin remodeling and genomic instability have been reported (19). miRNAs can regulate protein expression, and thus constitute potential miRNAs as therapeutic targets in cardiac and vascular disease, and can be used as novel biomarkers $(20,21)$. Likewise, it has been reported that cardiovascular diseases are closely related to the expression of circRNA, and they point to a high abundance of specific cardiac-expressed circRNA (9). The key cardiac genes include TTN, RYR2 and DMD in this study. In addition, it has been reported that lncRNA MALAT1, a highly abundant and conserved imprinted gene, has been implicated in many cardiovascular diseases; MALAT1 positively regulates the expression of Smad4 through sponging miR-204, and promotes osteogenic differentiation of human aortic valve interstitial cells (22).

We observed significant positive associations between circRNA and miRNA and the co-expression of circRNAmiRNA, but the causal relevance of these associations remains uncertain. In the study of $\mathrm{CHD}$, little research has been conducted on circRNAs, and especially the studies on the circRNA of coronary artery atherosclerosis are very few. Atherosclerosis is the main pathological basis of acute coronary syndrome. Thus, we aim to determine the positive associations between circRNA and CHD in a larger sample, and the positive co-expression between circRNA and miRNA in future investigations. Specifically, cell lines should be used to show that circRNA-miRNA mutually regulate 
the mechanism of mRNA, and a model animal should be established to confirm that circRNA/miRNA regulate mRNA and affect the formation and development of atherosclerosis.

In conclusion, the mechanism underlying CHD may be closely related to up- or downregulated circRNA and miRNA and co-expression of circRNA-miRNA specifically involved regulate multiple pathways and multiple cellular and molecular biological processes.

\section{Acknowledgements}

Not applicable.

\section{Funding}

This study was supported by the Foundation of Henan Educational Committee (grant nos. 18A320005 and 19A360032), and Beijing Medical and Health Foundation (grant no. B17246-045).

\section{Availability of data and materials}

The datasets used and/or analyzed during the current study are available from the corresponding author on reasonable request.

\section{Authors' contributions}

FLi was a major contributor in writing the manuscript, and responsible for conducting the experiments. GZhao contributed greatly in the designing of the study and was in main charge of the reviewing. ZC and XW acquired the data. FLv and $\mathrm{YZ}$ participated in the analysis and interpretation of the data. XY and WL designed the methods of this study. RC and $\mathrm{JL}$ were responsible for the follow-up of the patients. ML and GZhang participated in the design of the study. All authors read and approved the final manuscript.

\section{Ethics approval and consent to participate}

The study was approved by the Ethics Committee of the First Affiliated Hospital of Xinxiang Medical University (Xinxiang, China). Signed written informed consents were obtained from the patients and/or guardians.

\section{Patient consent for publication}

Not applicable.

\section{Competing interests}

The authors declare that they have no competing interests.

\section{References}

1. Jeck WR and Sharpless NE: Detecting and characterizing circular RNAs. Nat Biotechnol 32: 453-461, 2014.

2. Salzman J: Circular RNA Expression: Its potential regulation and function. Trends Genet 32: 309-316, 2016.

3. Qu S, Zhong Y, Shang R, Zhang X, Song W, Kjems J and Li H: The emerging landscape of circular RNA in life processes. RNA Biol 14: 992-999, 2017.
4. Tang CM, Zhang M, Huang L, Hu ZQ, Zhu JN, Xiao Z, Zhang Z, Lin QX, Zheng XL, Yang M, et al: CircRNA_000203 enhances the expression of fibrosis-associated genes by derepressing targets of miR-26b-5p, Colla2 and CTGF, in cardiac fibroblasts. Sci Rep 7: 40342, 2017.

5. Du WW, Yang W, Chen Y, Wu ZK, Foster FS, Yang Z, Li X and Yang BB: Foxo3 circular RNA promotes cardiac senescence by modulating multiple factors associated with stress and senescence responses. Eur Heart J 38: 1402-1412, 2017.

6. Li Y, Zheng Q, Bao C, Li S, Guo W, Zhao J, Chen D, Gu J, He X and Huang S: Circular RNA is enriched and stable in exosomes: A promising biomarker for cancer diagnosis. Cell Res 25: 981-984, 2015 .

7. Zhao Z, Li X, Gao C, Jian D, Hao P, Rao L and Li M: Peripheral blood circular RNA hsa_circ_0124644 can be used as a diagnostic biomarker of coronary artery disease. Sci Rep 7: 39918, 2017.

8. Fan X, Weng X, Zhao Y, Chen W, Gan T and Xu D: Circular RNAs in cardiovascular disease: An overview. BioMed Res Int 2017: 5135781, 2017.

9. Tan WL, Lim BT, Anene-Nzelu CG, Ackers-Johnson M, Dashi A, See K, Tiang Z, Lee DP, Chua WW, Luu TD, et al: A landscape of circular RNA expression in the human heart. Cardiovasc Res 113: 298-309, 2017.

10. You X, Vlatkovic I, Babic A, Will T, Epstein I, Tushev G, Akbalik G, Wang M, Glock C, Quedenau C, et al: Neural circular RNAs are derived from synaptic genes and regulated by development and plasticity. Nat Neurosci 18: 603-610, 2015.

11. Patterson TA, Lobenhofer EK, Fulmer-Smentek SB, Collins PJ, Chu TM, Bao W, Fang H, Kawasaki ES, Hager J, Tikhonova IR, et al: Performance comparison of one-color and two-color platforms within the MicroArray Quality Control (MAQC) project. Nat Biotechnol 24: 1140-1150, 2006.

12. Eisen MB, Spellman PT, Brown PO and Botstein D: Cluster analysis and display of genome-wide expression patterns. Proc Natl Acad Sci USA 95: 14863-14868, 1998.

13. Barabási AL and Oltvai ZN: Network biology: Understanding the cell's functional organization. Nat Rev Genet 5: 101-113, 2004.

14. Liu Y, Zheng L, Wang Q and $\mathrm{Hu}$ YW: Emerging roles and mechanisms of long noncoding RNAs in atherosclerosis. Int J Cardiol 228: 570-582, 2017.

15. Burd CE, Jeck WR, Liu Y, Sanoff HK, Wang Z and Sharpless NE: Expression of linear and novel circular forms of an INK4/ARFassociated non-coding RNA correlates with atherosclerosis risk. PLoS Genet 6: e1001233, 2010

16. Holdt LM, Beutner F, Scholz M, Gielen S, Gäbel G, Bergert H, Schuler G, Thiery J and Teupser D: ANRIL expression is associated with atherosclerosis risk at chromosome 9p21. Arterioscler Thromb Vasc Biol 30: 620-627, 2010.

17. Holdt LM, Stahringer A, Sass K, Pichler G, Kulak NA, Wilfert W, Kohlmaier A, Herbst A, Northoff BH, Nicolaou A, et al: Circular non-coding RNA ANRIL modulates ribosomal RNA maturation and atherosclerosis in humans. Nat Commun 7: 12429, 2016.

18. Wang K, Long B, Liu F, Wang JX, Liu CY, Zhao B, Zhou LY, Sun T, Wang M, Yu T, et al: A circular RNA protects the heart from pathological hypertrophy and heart failure by targeting miR-223. Eur Heart J 37: 2602-2611, 2016.

19. Siede D, Rapti K, Gorska AA, Katus HA, Altmüller J, Boeckel JN, Meder B, Maack C, Völkers M, Müller OJ, et al: Identification of circular RNAs with host gene-independent expression in human model systems for cardiac differentiation and disease. J Mol Cell Cardiol 109: 48-56, 2017.

20. Lei W, Feng T, Fang X, Yu Y, Yang J, Zhao ZA, Liu J, Shen Z, Deng $\mathrm{W}$ and $\mathrm{Hu} \mathrm{S}$ : Signature of circular RNAs in human induced pluripotent stem cells and derived cardiomyocytes. Stem Cell Res Ther 9: 56, 2018.

21. Vegter EL, Schmitter D, Hagemeijer Y, Ovchinnikova ES, van der Harst P, Teerlink JR, O'Connor CM, Metra M, Davison BA, Bloomfield D, et al: Use of biomarkers to establish potential role and function of circulating microRNAs in acute heart failure. Int $\mathrm{J}$ Cardiol 224: 231-239, 2016.

22. Xiao X, Zhou T, Guo S, Guo C, Zhang Q, Dong N and Wang Y: LncRNA MALAT1 sponges miR-204 to promote osteoblast differentiation of human aortic valve interstitial cells through up-regulating Smad4. Int J Cardiol 243: 404-412, 2017.

This work is licensed under a Creative Commons Attribution-NonCommercial-NoDerivatives 4.0 International (CC BY-NC-ND 4.0) License. 